Reprod. Nutr. Dévelop., 1984, 24 (2), 197-206.

\title{
Immunité chez le fœtus et le nouveau-né : modèle porcin
}

\author{
H. SALMON
}

\begin{abstract}
Laboratoire de Pathologie porcine, I.N.R.A., Groupe de Laboratoires de Pathologie animale de Thiverval-Grignon, 78850 Thiverval-Grignon, France.
\end{abstract}

Summary. Immunity of the fetus and the newborn: a porcine model.

An efficient placental barrier protects the pig fetus from all exogenous antigenic stimulation. The results of studies on different subpopulations of lymphocytes, histological localization, and stimulation as well as immunization in utero with allogeneic lymphocytes have confirmed that the pig fetus becomes immunocompetent at around 80 days of fetal life (total length of gestation : 115 days).

Because the newborn piglet has had no " immunological experience " in utero, it can only elaborate a primary response which is often advantageous to pathogenic microorganisms. Under natural conditions, immune protection is provided by the mother in the form of colostrum. This colostrum provides systemic immunity and is followed by milk which insures local immunity in the piglet gastrointestinal tract. However, the great disadvantage of maternal antibodies is that they suppress the active synthesis of new antibodies in young pig. Thus. the beginning of active immunity in the piglet is a function of the ratio of maternal antibody level to the level of antigenic stimulation.

\section{Introduction.}

La notion familière que le nouveau-né est très sensible aux infections par suite de son immaturité immunologique (c'est-à-dire qu'il est plus ou moins apte à répondre immunologiquement contre divers agents pathogènes) se devait d'être vérifiée expérimentalement, et éprouvée vis-à-vis d'une autre hypothèse mettant en relief le fait que le nouveau-né manque d'expérience immunologique, c'est-àdire qu'il est vierge de stimulation antigénique antérieure.

Cette virginité antigénique dépend en fait du nombre de couches anatomiques séparant le fœetus de sa mère; plus le nombre de couches est important moins le placenta sera perméable aux antigènes et anticorps provenant de la mère.

Parmi les différentes espèces de mammifères, ce sont les espèces équine et porcine qui présentent la barrière placentaire la plus efficace (Tizar, 1982). Ces espèces représentent ainsi un modèle d'étude du développement de la réponse immunitaire chez le fœetus en l'absence d'anticorps (Kim et al., 1966; Sterzl et 
Silverstein, 1967) ou de stimulation antigénique d'origine maternelle quoique ce dernier point se vérifie indirectement.

Au surplus le porc représente un matériel unique d'étude du développement de la réponse immunitaire, dans la mesure où il peut être séparé de sa mère et élevé dans un environnement stérile, avec une alimentation non-antigénique ; ces études permettront de préciser le rôle respectif des anticorps maternels et de l'antigène, sur la mise en route de la réponse immunitaire propre du porcelet nouveau-né.

\section{Mise en évidence de l'immuno-compétence du fœtus}

A. Chronologie d'apparition des cellules du système immunitaire.

Les données récentes de l'immunologie montrent que les réactions immunitaires mettent en jeu au moins trois types de cellules, macrophages, lymphocytes $T$ et lymphocytes B avec pour chacun des types des sous-classes qu'on peut identifier par des marqueurs de membrane qui dans certains cas sont eux-mêmes des marqueurs de fonction (Salmon, 1979).

1) Le système réticulo-endothélial. - Ce système comprend les macrophages qui sont localisés dans des positions stratégiques pour éliminer les substances étrangères du sang. La capacité de phagocytose commence très précocément chez le fœetus du porc (Solomon, 1971).

2) Lymphocytopoïèse. - D'après Solomon (1971) le démarrage de l'immuno-compétence du fœetus coïncide avec le moment où les petits lymphocytes apparaissent dans le sang (" périphéralisation »).

A la lumière des données actuelles de l'immunologie on peut interpréter cette " périphéralisation » comme une nécessité pour les lymphocytes $T$ helper d'aller au contact des lymphocytes B sensibilisés à l'antigène, pour les aider à synthétiser des anticorps, au lieu d'être rendus tolérants. Cependant ces données morphologiques (présence des lymphocytes ou de leurs sous-populations) doivent être étayées par la mise en évidence des fonctions soit in vitro soit in vivo.

a) Apparition des lymphocytes et périphéralisation. - Les premiers lymphocytes reconnaissables morphologiquement apparaissent dans le thymus (J-28) puis dans la rate $(\mathrm{J}-48)$ et les ganglions lymphatiques $(\mathrm{J}-52)$ (Sterzl et Kovaru, 1977). On trouve également des lymphocytes dans l'intestin dès le $50^{\mathrm{e}}$ jour, disséminés dans l'épithélium (Salmon, résultats non publiés), et associés sous forme d'agrégats, représentant des ébauches de Plaque de Peyer (Chapman et al., 1974).

b) Apparition des sous-populations /ymphocytaires.

- Apparition des lymphocytes T: Le premier organe où apparaissent des lymphocytes $\mathrm{T}$ (détectés par la présence du récepteur aux globules rouges de mouton ou d'antigène $T$ spécifique) est le thymus (J-38) (Kovaru et Jaraskova, 1978). Puis les lymphocytes vont émigrer pour coloniser les organes lymphoïdes 
dits secondaires ; le moment exact de colonisation par les premiers lymphocytes $\mathrm{T}$ est difficile à déterminer car le phénomène est progressif. Néanmoins on peut affirmer qu'à $\mathrm{J}-50$, on trouve des lymphocytes $T$ dans tous les organes étudiés (Salmon, 1982).

- Apparition des /ymphocytes B: La présence de précurseurs des cellules B (cellules comportant des chaînes $\mu$ intracytoplasmiques, mais ne présentant pas d'immunoglobines membranaires) a été mise en évidence dans la rate de foetus à J-55 (Chapman et al., 1974). Cependant, Jaraskova et al. (1978) ont mis en évidence des lymphocytes $B$ à lgM membranaire dès le $44^{e}$ jour dans le foie fœtal puis dans la rate $(\mathrm{J}-51)$ et enfin dans la moelle osseuse $(\mathrm{J}-60)$. Ces derniers résultats semblent donc exclure l'hypothèse suivant laquelle, les plaques de Peyer seraient l'équivalent de la bourse de Fabricius des oiseaux, puisque ces dernières ne sont occupées par les lymphocytes qu'au 50 jour.

c) Détermination de la période où la proportion des sous-classes de lymphocytes est la même que chez l'adulte. - Au lieu de déterminer le moment où apparaissent les premiers lymphocytes à la périphérie, ce qui est somme toute très difficile à définir avec exactitude, on peut s'attacher à déterminer le moment où la proportion observée des différentes sous-classes est la même que chez l'adulte, en partant de l'hypothèse que cette proportion atteinte, signe l'immunocompétence. Quelle que soit la sous-population envisagée : T, B, L ou Nulle (Salmon, 1979), le sang et le thymus présentent dès le $80^{\circ}$ jour de gestation un profil adulte, l'intestin et le ganglion mésentérique seulement à la naissance ; la rate est le seul organe qui à la naissance ne présente pas le type adulte ; cependant ce retard est comblé 4 jours après (Salmon, 1982).

3) Chronologie d'apparition des structures histologiques spécifiques des organes lymphoïdes (Salmon, 1982). - Une bonne réponse immunitaire nécessite que les différentes classes de lymphocytes puissent se développer et coopérer dans un environnement adéquat, représenté par des structures spécifiques des organes lymphoïdes. Les principales étapes du développement des structures lymphoïdes caractéristiques de chacun des organes sont les suivantes:

- Thymus : démarcation entre le cortex et la medulla, dès le $50^{\mathrm{e}}$ jour de la gestation.

- Ganglion mésentérique : Le cortex s'isole de la medulla au $80^{\mathrm{e}}$ jour et on note la présence de veinules post-capillaires dans le cortex, suggérant la possibilité, déjà à cette période, d'une recirculation des lymphocytes.

On note la présence de quelques follicules primaires (zone B) seulement à la naissance.

- Intestin : Dès le $50^{\mathrm{e}}$ jour, les lymphocytes sont présents dans l'épithélium et la lamina propria.

Les plaques de Peyer sont visibles à l'œil nu au $80^{e}$ jour, mais les zones thymo-dépendantes (interfolliculaires) sont peu développées; au contraire du ganglion mésentérique on note la présence de nombreux follicules (zone B). 
- Rate : La pulpe rouge se développe avant la pulpe blanche; cette dernière n'apparaît qu'au $105^{\mathrm{e}}$ jour, et s'accroît surtout après la naissance. Elle revêt la forme classique de zone périartériolaire : cependant on ne note pas la présence de corpuscule de Malpighi (zone B). En général il y a absence de centre germinatif et de plasmocytes confirmant que le fœetus de porc se développe dans un environnement vierge de toute stimulation antigénique.

\section{B. Détermination du moment où le fotus de porc est immunocompétent.}

Nous avons vu que les sous-populations lymphocytaires et les macrophages sont présents très précocément dans le fœetus de porc autour du $60^{\mathrm{e}}$ jour de vie fœetale. Cependant pour qu'un lymphocyte soit immunocompétent il faut qu'il possède les récepteurs à l'antigène et qu'il soit inductible par cet antigène.

D'un point de vue fondamental la chronologie d'apparition des récepteurs à différents antigènes est destinée à vérifier la théorie de diversification du répertoire de reconnaissance de l'antigène. Chez le porc les expérimentateurs se sont bornés à montrer l'existence d'un état inductible, qui prouve a posteriori la présence de récepteurs.

Cette induction est démontrée par la présence de cellules terminales fonctionnelles, soit in vitro après cultures des cellules avec l'antigène, soit in vivo après injection de l'antigène chez le fœtus.

\section{Détermination de l'immunocompétence en culture de tissu.}

a) Réponse spécifique. - Par la technique de culture des cellules en présence d'antigène et révélation des cellules secrétant les anticorps (PFC) on met en évidence des PFC dans le foie au 24 jour de vie fotale (45 PFC $/ 10^{8}$ cellules), puis au $35^{\mathrm{e}}$ jour dans le thymus et au $69^{\mathrm{e}}$ jour dans la rate et le ganglion lymphatique (Sterzl et Kovaru, 1977 ; Schultz et al., 1971). Comme les récepteurs immunoglobuliniques ne sont pas détectés avant le $50^{\circ}$ jour, ces résultats suggèrent que la densité des lgb de surface sur des lymphocytes hépatiques est très faible ou encore qu'à cette époque le récepteur n'est pas une molécule complète d'anticorps.

b) Réponse non spécifique aux mitogènes. - A J-60 les thymocytes répondent à la stimulation par les mitogènes (PHA, Con A, PMW, Soybean), mais également les lymphocytes du sang; les lymphocytes de la rate sont stimulés plus tardivement vers J-90, en accord avec les résultats de Rodey et al. (1972) ; cependant concomitamment à une augmentation de réponse aux mitogènes des cellules spléniques, nous observons une diminution de réponse des thymocytes (Salmon, résultats non publiés).

\section{Détermination de l'immunocompétence in vivo.}

Quel que soit l'antigène envisagé, le développement de la réponse immunitaire peut être envisagée comme une transition de l'état de tolérance à l'induction de l'immunité. 
a) Tolérance et immunité de transplantation. - A différentes périodes de la vie fœtale (de J-60 à J-80) des fœetus reçoivent par injection des cellules allogéniques, provenant de donneurs non apparentés (Binns, 1967). D'après le devenir d'une greffe de peau du donneur sur les nouveaux-nés, on en déduit l'état du système immunitaire au moment de l'injection des cellules allogéniques.

Lorsque les nouveaux-nés ont reçu les cellules allogéniques à J-60, ils ne rejettent pas la greffe cutanée; en revanche ceux injectés plus tard $(\mathrm{J}-80)$ sont capables de rejeter la greffe cutanée.

Ainsi la tolérance aux cellules allogéniques peut être induite à J-60 et disparaît pour faire place à une immunité de transplantation à $\mathrm{J}-80$.

b) Réponse anticorps. - Le moment où le fœetus de porc semble être capable de répondre par la formation d'anticorps sériques semble être le $70^{\mathrm{e}}$ jour de vie foetale ; l'injection de phage $\phi \times 174$ au $70^{\mathrm{e}}$ jour par la veine ombilicale est suivie d'une réponse anticorps d'autant plus précoce que la dose de phage injectée est plus importante (Hajek et al., 1969).

Par la suite le foetus est capable de répondre chronologiquement d'abord aux parvovirus $(\mathrm{J}-72)$, à $E$. rhusopathiae $(\mathrm{J}-73)$ et à $(\mathrm{J}-80)$ à $S$. orianenberg (cité par Tizar, 1982). Les anticorps formés sont de type IgM. L'équipe tchèque (Sterzl et Kovaru, 1977) a pu montrer que les changements cytologiques dans le fœetus de porc dépendent du niveau de développement atteint par le tissu lymphatique au moment de l'immunisation. Ainsi l'injection de globules rouges de mouton à J-70 (caractérisée par l'absence de structure folliculaire), n'est pas suivie d'apparition de plasmocytes ; en revanche l'injection à $J-83$ loù les structures folliculaires existent) est suivie d'une apparition de plasmocytes dans la rate et le sang, ce nombre augmentant avec celui des globules rouges injectés.

c) Locale. - Normalement l'intestin et le poumon du fœtus devraient être la route principale de stimulus dérivé de n'importe quel antigène qui peut envahir le sanctuaire stérile de l'utérus et faire partie du fluide amniotique. Des études (Husband et Mc Dowell, 1975) ont montré que des antigènes introduits directement dans la cavité amniotique de brebis gestante peuvent conduire à une immunisation orale du foetus avec développement de plasmocytes synthétisant les anticorps IgM dans l'intestin.

Chez le porc l'injection de virus TGE par voie intramusculaire (Redman et al., 1978) est suivie d'une localisation et multiplication du virus dans l'intestin fœtal ; la destruction des villosités est vite régénérée mais déjà au $74^{\mathrm{e}}$ jour d'infection le foetus a élaboré des anticorps des 3 classes, G, A, M. Dans certains cas on peut même assister à une protection ultérieure du porcelet nouveau-né à une épreuve virulente, et sans production de réponse anticorps anamnestique.

Ces résultats prouvent bien l'existence de l'immunocompétence fœtale et la possibilité de vacciner le fœus in utero, possibilité également éprouvée chez les bovins avec $E$. Coli et les réovirus (Conner et Carter, 1975).

d) Les immunoglobulines fotales. - La détection d'immunoglobulines des classes lgG et $\lg A$ (cette dernière 25 à 50 fois moindre que les lgG) chez le fotus, l'absence d'lgM (Prokesova et al., 1977) ou même des 3 classes (Franz et al., 
1982) ne remet pas en question le fait que le fœtus de porc se développe à l'abri de stimulus antigénique et des immunoglobulines maternelles.

Ces immunoglobulines sont synthétisées par les tissus fœtaux en culture et peuvent correspondre à la libération d'immunoglobulines membranaires (Hampl et al., 1980). Comme la proportion des différentes sous-classes est différente au niveau des cellules et du sérum, la prédominance d'une des sous-classes dans le sérum reflète une libération plus rapide de ce type d'immunoglobuline.

Au surplus les IgG fœtales sont très hétérogènes en comparaison de celles de l'adulte, pouvant recouvrir les différents fragments de la molécule entière (Prokesova et al., 1977).

\section{Développement post-natal}

Vierge de toute expérience immunitaire avant la naissance, mais cependant immunocompétent, le nouveau-né, dans les conditions d'élevage conventionnel, est brusquement mis au contact d'un grand nombre de déterminants antigéniques comme conséquence de l'établissement de la respiration, de la colonisation de l'intestin par une flore et de l'absorption de matériel antigénique trouvé dans l'alimentation. C'est l'exposition à ces antigènes complexes et multiples qui cause la maturation du tissu lymphoïde (apparition de plasmocytes, activité folliculaire), l'apparition spontanée d'anticorps d'un certain type, et surtout l'augmentation du nombre de cellules immunologiquement compétentes capables de réagir rapidement à une réinjection de l'antigène, tous ces processus n'intervenant pas en l'absence de stimuli antigéniques ainsi que l'ont montré les expérimentations sur porcelet axénique (Kim et al., 1979), n'ayant absorbé ni colostrum, ni lait.

Dans le cas où l'exposition du nouveau-né est suivie de réponse immune (voir ci-dessous), la réponse est de type primaire (à cause de l'absence d'expérience immunologique in utero), c'est-à-dire à démarrage lent. Ainsi si l'antigène est représenté par un agent pathogène, ce dernier profite du laps de temps pour se multiplier et se disséminer dans l'organisme ; le même phénomène s'observe chez un porc adulte élevé dans des conditions axéniques, placé soudainement dans un environnement normalement " infecté ». Heureusement, dans les conditions naturelles, le nouveau-né reçoit de sa mère par le colostrum et le lait une couverture immunitaire efficace, qui cependant peut avoir un effet modulateur sur sa réponse immunitaire propre.

\section{A. Effet des anticorps maternels pour le nouveau-né.}

1) Effet protecteur.

a) Immunité systémique. - Comme aucune transmission d'anticorps n'a pu s'opérer durant la vie fotale et que l'intestin du porcelet n'est perméable que les 12 à $36 \mathrm{~h}$ après la naissance (chez les rongeurs 21 jours !), le porcelet reçoit un concentré d'immunoglobulines plasmatiques (la proportion relative des 3 classes $G$, A, $M$ est la même que dans le sérum), sous forme de colostrum (Tizar, 1982).

Quoique la proportion d'lgG soit prédominante (85\%), celles des $\lg A$ et $\lg M$ ne représentant que $15 \%$, il ne faudrait pas en conclure que seules les lgG sont 
indispensables pour là protection du nouveau-né. Si les lgG peuvent neutraliser les entérotoxines (Brandenberg et Wilson, 1973), des IgM anti-E. Coli sont beaucoup plus à même d'exercer in vivo une élimination bactérienne (Childlow et Porter, 1978).

Le rôle de couverture immunitaire par la mère est bien mis en évidence par l'observation qu'E. Coli ne se multiplie dans l'intestin de truie gestante qu'en fin de gestation, amenant ainsi la production d'IgM anti-E. Coli (Porter, 1979), passant dans le colostrum, et ayant un rôle protecteur beaucoup plus important que celui des $\operatorname{lgG}$.

b) Immunité locale. - Lorsque le tube digestif du porcelet devient imperméable au passage des anticorps colostraux, le colostrum fait place au lait : le lait de truie est particulièrement riche en lgA secrétées localement par la mamelle (Bourne et Curtis, 1973). Ces IgA ne sont pas absorbées par l'intestin, elles restent fixées au niveau de l'épithélium et assurent la protection locale du porcelet contre les entérites digestives (entérites impliquant des entérocytes villeux par opposition à celles impliquant les entérocytes des cryptes), mieux que toutes les autres classes d'lgG (Bohl et al., 1972).

2) Effet régulateur des anticorps maternels sur la réponse immunitaire propre du porcelet. - Depuis Uhr et Moller (1968), on sait que des anticorps administrés passivement chez les rongeurs peuvent avoir un effet régulateur sur la réponse immunitaire.

Ainsi l'administration d'anticorps avant ou peu après l'injection d'antigène supprime la réponse primaire.

Chez le porc nouveau-né les anticorps colostraux inhibent l'expansion des clônes des lymphocytes $B$ correspondant à l'antigène par un mécanisme d'élimination antigénique (Muscoplat et al., 1977), ou encore en se liant au récepteur Fc des lymphocytes T. (Setcavage et Kim, 1980). Cependant il apparaît que dans les conditions d'immunisation du porcelet sous la mère, c'est le rapport antigèneanticorps maternel qui détermine de niveau de réponse du porcelet (Launais et al., 1978) et que dans certaines circonstances des $\lg M$ peuvent augmenter une réponse primaire au lieu de la supprimer (Henry et Jerne, 1968). Enfin la réponse primaire peut ne pas s'extérioriser, mais le stimulus antigénique est enregistré et ultérieurement on assistera à une réponse d'emblée de type secondaire (Launais et al., 1978 ; Thalhammer et al., 1978).

\section{B. Mise en route de l'immunité active du porcelet sous la mère.}

1) Immunité systémique. - Les immunoglobulines maternelles colostrales seront progressivement éliminées du porcelet par son catabolisme (environ 15 jours pour dégrader la moitié des $\lg$, 3 jours pour des $\lg M$ ) et remplacées par des immunoglobulines propres du porcelet (Metzger et al., 1982).

2) Immunité locale intestinale. - Comme lors d'immunisation par voie générale, la réponse primaire immune intestinale contre la colonisation microbienne ou l'administration orale d'antigènes bactériens, est dominée par la production $\mathrm{d}^{\prime} \mathrm{lg} \mathrm{M}$, puis progressivement les plasmocytes à lgM laissent la place aux plasmocytes à IgA (Allen et Porter, 1977). 
La production d'lgM représente cependant un mécanisme de défense efficace de la muqueuse intestinale ; en raison de la faible liaison entre lgM et composant secrétoire, les IgM sont libérées facilement dans la barrière intestinale : elles auraient là pour rôle soit d'agglutiner les germes pathogènes qui seraient rejetés ultérieurement grâce au péristaltisme intestinal, soit encore d'opsoniser des germes qui pourraient être phagocytés par les polynucléaires présents dans l'intestin (Bellamy et Nielsen, 1974).

\section{Conclusions et perspectives}

Les données de l'ontogénie confirment que le porc a acquis son immunocompétence avant la naissance. Cependant, quoique capable de répondre à une stimulation antigénique, le porcelet nouveau-né élabore un type de réponse primaire. Dans les conditions naturelles une couverture immunitaire lui est apportée par sa mère via la prise de colostrum et de lait.

Pratiquement on peut envisager d'augmenter le niveau d'anticorps spécifiques dans le colostrum et le lait par la vaccination appropriée de la mère, ou d'augmenter l'expérience immunologique du porcelet par une vaccination de ce dernier. Dans ce cas, il sera bon de se souvenir que la présence des anticorps passifs maternels (exception faite de l'immunisation in utero) peut interférer avec l'immunisation active du porcelet. Des études appropriées à chaque cas sont nécessaires pour juger de la dose d'antigène à injecter, de la voie d'administration en rapport avec le niveau et la classe d'immunoglobuline et de l'utilisation d'un adjuvant. Ce type de vaccination est le seul envisageable lorsqu'un germe pathogène septicémique envahit la porcherie :

1) Au moment de la mise-bas, les anticorps colostraux n'ayant plus le temps d'être synthétisés donc d'intervenir ;

2) A partir de la $2^{\mathrm{e}}$ semaine lorsque le taux des anticorps maternels sériques est très fortement diminué ;

3) Après le sevrage lorsque les anticorps contenus dans le lait ne sont plus donnés au porcelet.

Pour ce dernier cas, le porcelet a pris suffisamment d'âge pour être moins sensible aux conséquences néfastes d'un germe pathogène, et il s'immunisera activement pour acquérir son expérience immunologique.

9. Réunion du groupe Développement I.N.R.A., Rennes, 4-5 mai 1983.

\section{Références}

ALLEN W. D., PORTER P., 1977. The relative frequencies and distribution of immunoglobulinbearing cells in the intestinal mucosa of neonatal and weaned pigs and their significance in the development of secretory immunity. Immunology, 32, 819-824.

BELLAMY J. E., NIELSEN N. O., 1974. Immune mediated emigration of neutrophils into the lumen of the small intestine. Infect. Immunol., 9, 615-619. 
BINNS R. M., 1967. Bone-marrow and lymphoïd cell injection of the pig foetus resulting in transplantation tolerance or immunity and immunoglobulin production. Nature, 214, 179-181.

BOHL E. H., GUPTA R. K. P., OLQUIN F. M. W., SAIF L. J., 1972. Antibody response in serum, colostrum and milk of swine after infection or vaccination with transmissible gastroenteritis virus. Infect. Immunol., 6, 289-301.

BOURNE F. J., CURTIS J., 1973. The transfer of immunoglobulins IgG, IgA, and IgM from serum to colostrum and milk in the sow. Immunology, 24, 157-162.

BRANDENBERG A. C., WILSON M. R., 1973. Immunity to $E$. Coli in pigs : IgG immunoglobulin in passive immunity to $E$. Coli enteritis. Immunology, 24, 119-127.

CHAPMAN H. A., JOHNSON J. S., COOPER M. D., 1974. Ontogeny of Peyer's patches and immunoglobulin-containing cells in pigs. J. Immunol., 112, 555-562.

CHIDLOW J. W., PORTER P., 1978. The role of oral immunization in stimulating $E$. Coli antibody of the IgM class in porcine colostrum. Res. vet. Sci, 24, 254-257.

CONNER G. H., CARTER G. R., 1975. Response of the bovine foetus to reovirus. Vet. med. small Anim. Cli, 70, 1463-1464.

FRANZ J., MILON A., SALMON H., 1982. Synthesis of immunoglobulins $\operatorname{lgG}$, $\lg M$ and $\lg A$ during the ontogeny of fœtal pigs. Acta vet. (Brno), 51, 23-31.

HAJEK P., KOVARU F., KRUML J., 1969. The antibody response and morphological changes following immunization of pig foetuses with $\Phi \times 174$ phage. Fol. microbiol. (Praha), 14, 492.

HAMPL J., FRANZ J., STEPANEK J., HOLUB A., MENSIK J., 1980. Immunoglobulin proteosynthesis in organ cultures from newborn piglets. Acta vet. (Brno), 49, 67-74.

HENRY C., JERNE N. K., 1968. Competition of 19S and 7S antigen receptors in the regulation of the primary immune response. J. exp. Med., 128, 133-152.

HUSBAND A. J., Mc DOWELL G. H., 1975. Local and systemic immune responses following oral immunization of foetal lambs. Immunology, 29, 1019-1028.

JARASKOVA L., TREBICHAVSKY I., KOVARU F., PROKESOVA L., 1978. Precursors of B-lymphocytes with surface $\operatorname{lgM}$ and their possible migration from bone-marrow to peripheral lymphatic tissues. Lymphology, 11, 81-83.

KIM Y. B., BRADLEY S. G., WATSON D. W., 1966. Antibody synthesis in germ-free colostrumdeprived miniature piglets, 273-284. In BUSTAD L. K., Mc CELLAN R. O. Swine in biomedical research. Battelle Memorial Institute, Pacific Northwest Labor., Richmond, Washington.

KIM Y. B., SeTCAVAGe T. M., KIM D. J., CHUN H. G., SCHEFFEL J. W., 1979. Ontogenic development and differenciation of the immune system in the gnotobiotic miniature swine, 203-213. In FLIEDNER et al., Clin. experim. Gnotobiotics, Zbl. Bakt. Suppl. 7, Gustav. Fischer Verlag-Stuttgart, New-York.

KOVARU F., JARASKOVA L., 1978. Development of E-rosette formation in ontogeny of pigs. Fol. biol. (Praha), 24, 399-400.

LAUNAIS M., AYNAUD J. M., CORTHIER G., 1978. Hog cholera virus : active immuzation of piglets with the Thiversal strain in the presence and absence of colostral passive immunity. Vet. Microbiol., 3, 31-43.

METZGER J. J., SALMON H., MILON A., 1982. Immunologie, 129-147. In MORNET P., TOURNUT J., TOMA B. Le Porc, Maloine éd., Paris.

MUSCOPLAT C. C., SETCAVAGE T. M., KIM Y. B., 1977. Regulation of the immune response in neonatal piglets by maternal antibody. Int. Arch. Allergy appl. Immun., 54, 165-170.

PORTER P., 1979. Structural and functional characteristic of immunoglobulins of the common domestic species. Adv. vet. Sci. comp. Med., 23, 1-21.

PROKESOVA L., REJNEK J., STERZL L., 1977. The immunoglobulins and antibody activity in sera of pig foetuses and colostrum-deprived newborns. Acta vet. (Brno), 46, suppl. 4, 83-118.

REDMAN R. D., BOHL E. H., CROSS R. F., 1978. Intrafoetal inoculation of swine with transmissible gastroenteritis virus. Am. J. Vet. Res., 39, 907-911.

RODEY G. E., DAY N., HOLMES-GRAY B., GOOD R. A., 1972. Ontogeny of the phytohemagglutinin response of fetal piglet thymic and splenic lymphocytes. Infect. Immunity, 5, 337-338.

SALMON H., 1979. Surface markers of porcine lymphocytes and distribution in various lymphoïd organs. Int. Arch. Allergy appl. Immun., 60, 262-274.

SALMON H., 1982. Ontogénie des sous-populations lymphocytaires dans les différents organes du porc fœtal, nouveau-né et jeune adulte, 43-54. 6e Sém. GTV-INRA, Versailles. 
SCHULTZ R. D., WANG M. S., DUNNE H. W., 1971. Development of the humoral immune response of the pig. Am. J. vet. Res., 32, 1331-1336.

SETCAVAGE T. M., KIM Y. B., 1980. Inhibition of the immune response by Fc receptor-bound specific antibody on T lymphocytes. J. Immunol., 124, 553-556.

SOLOMON J. B., 1971. Foetal and neonatal immunology. North-Holland, Amsterdam.

STERZL J., KOVARU F., 1977. Development of lymphatic tissue and immunocompetency in pig foetuses and germ-free piglets. Acta vet. (Brno), 46, suppl. 4, 13-53.

STERZL J., SILVERSTEIN A. M., 1967. Developmental aspects of immunity. Adv. Immunol., 6. 337-459.

THALHAMMER J. G., STOCKL W., REYERO C., 1978. Effects of the B-cell activators lipid A and dextran sulphate on the antibody response to sheep red blood cells in piglets. Immunology, 35. 793-799.

TIZAR I., 1982. An introduction to veterinary immunology. Saunders W. B. Philadelphia.

UHR J. W., MOLLER G., 1968. Regulatory effect of antibody on the immune response. Adv. Immunol., 8, 81-127. 